\title{
3-Ketosteroid 9 $\alpha$-hydroxylase enzymes: Rieske non-heme monooxygenases essential for bacterial steroid degradation
}

\author{
Mirjan Petrusma $\cdot$ Robert van der Geize • \\ Lubbert Dijkhuizen
}

Received: 30 January 2014 / Accepted: 25 April 2014/Published online: 21 May 2014

(C) The Author(s) 2014. This article is published with open access at Springerlink.com

\begin{abstract}
Various micro-organisms are able to use sterols/steroids as carbon- and energy sources for growth. 3-Ketosteroid 9 $\alpha$-hydroxylase (KSH), a two component Rieske non-heme monooxygenase comprised of the oxygenase KshA and the reductase KshB, is a key-enzyme in bacterial steroid degradation. It initiates opening of the steroid polycyclic ring structure. The enzyme has industrial relevance in the synthesis of pharmaceutical steroids. Deletion of KSH activity in sterol degrading bacteria results in blockage of steroid ring opening and is used to produce valuable C19-steroids such as 4-androstene-3,17-dione and 1,4androstadiene-3,17-dione. Interestingly, $\mathrm{KSH}$ activity is essential for the pathogenicity of Mycobacterium tuberculosis. Detailed information about KSH thus is of medical relevance, and KSH inhibitory compounds may find application in combatting tuberculosis. In recent years, the 3D structure of the KshA protein of M. tuberculosis $\mathrm{H} 37 \mathrm{Rv}$ has been elucidated and various studies report biochemical characteristics and possible physiological roles of KSH. The current knowledge is reviewed here and forms a solid basis for
\end{abstract}

M. Petrusma · R. van der Geize · L. Dijkhuizen ( $\square)$ Department of Microbiology, Groningen Biomolecular Sciences and Biotechnology Institute (GBB), University of Groningen, Nijenborgh 7, 9747 AG Groningen,

The Netherlands

e-mail: L.Dijkhuizen@rug.nl

M. Petrusma

e-mail: j.m.petrusma@rug.nl further studies on this highly interesting enzyme. Future work may result in the construction of KSH mutants capable of production of specific bioactive steroids. Furthermore, KSH provides an promising target for drugs against the pathogenic agent $M$. tuberculosis.

Keywords 3-Ketosteroid 9 $\alpha$-hydroxylase . Rieske mono-oxygenase $\cdot 9 \alpha$-Hydroxylation . Rhodococcus $\cdot$ Mycobacterium $\cdot$ Steroid biotransformation

\section{Introduction}

Early observations that microbes are able to degrade cholesterol were made by Söhngen (1913). Since then, a range of bacteria were found to be able to utilize sterols (Arima et al. 1969; Nagasawa et al. 1969), many of which are actinobacteria (e.g. Rhodococcus, Mycobacterium) (Donova 2007; Fernandes et al. 2003; Malaviya and Gomes 2008 (reviews)). Various enzyme steps in the bacterial sterol degradation pathways remain to be characterized, but in recent years knowledge on sterol degradation has greatly increased. A cholesterol catabolism gene cluster was identified in $R$. jostii RHA1 and a cholesterol degradation pathway was predicted (Van der Geize et al. 2007) (Fig. 1). Cholesterol is a C27 sterol and degradation of its side chain to a $\mathrm{C} 22$ intermediate occurs by a process similar to $\beta$-oxidation of fatty 
A

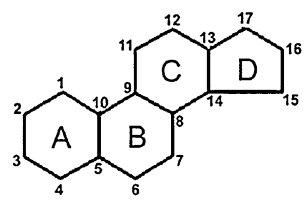

\section{B}

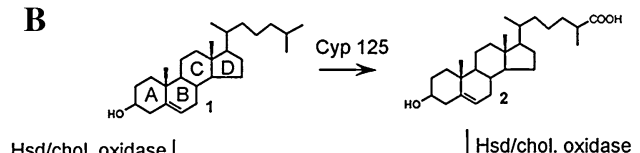

Hsd/chol. oxidase $\quad$ Hsd/chol. oxidase

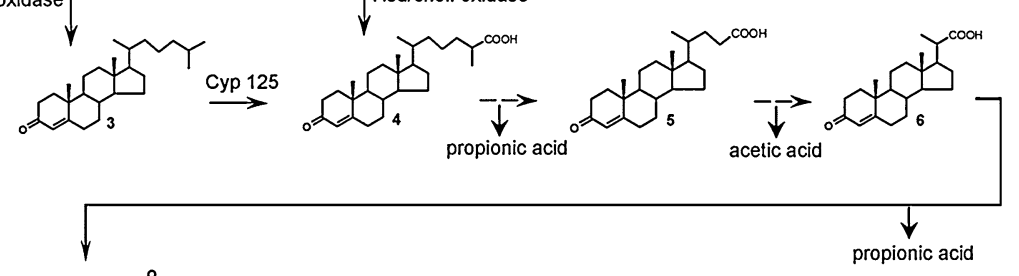

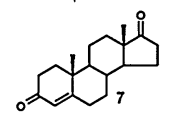
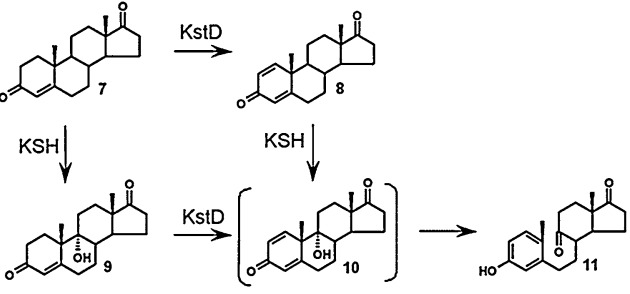

$\stackrel{\mathrm{HsaAB}}{\longrightarrow}$
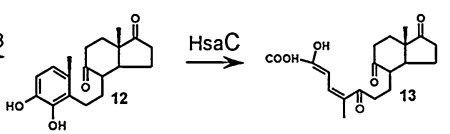

$\stackrel{\text { HsaD }}{\longrightarrow}$

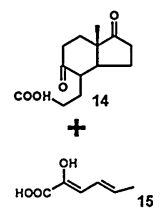

Fig. 1 a The basic polycyclic ring structure of sterols and steroids with carbon atoms 1-17. b Proposed cholesterol catabolism in Rhodococcus species and Mycobacterium tuberculosis (Adapted from Van der Geize et al. 2007). Dashed arrows indicate multiple enzymatic steps. The depicted steroids are 1 5-cholestene-3 $\beta$-ol (cholesterol), 2 5-cholestene-26-oic acid-3 $\beta$-ol, 3 4-cholestene-3-one, 4 4-cholestene-26oic acid-3one, 5 4-cholestene-24oic acid-3-one, 6 3-oxo-23,24-bisnorchola-4-ene-22-oic acid, 7 4-androstene-3,17-dione (AD), 81 , 4-androstadiene-3,17-dione (ADD), $99 \alpha$-hydroxy-4-androstene-3,17-dione (9OHAD), $109 \alpha$-hydroxy-1,4-androstadiene3,17-dione (ADD), 11 3-hydroxy-9,10-secoandrost-1,3,5(10)triene-9,17-dione (3-HSA), 12 3,4-dihydroxy-9,10-secoandrost1,3,5(10)-triene-9,17-dione (3,4-DHSA), 13 4,5-9,10-diseco-3-

acids, yielding propionic acid and acetic acid. The subsequent formation of C19 steroids yields another propionic acid (Sih et al. 1968). In M. tuberculosis, metabolic labeling studies at carbon atom $\mathrm{C} 26$ showed that the propionic acid released from the side chain is a precursor in the production of phthiocerol dimycocerosate (PDIM), a surface lipid (Ouellet et al. 2010). The $\mathrm{C} 19$ central metabolite derived from cholesterol is $\mathrm{AD}$, containing the polycyclic ring structure. Steroid ring degradation has been described in some detail for the Gram-negative bacterium Comamonas testosteroni TA441 (Horinouchi et al. 2012) and for the hydroxy-5,9,17-trioxoandrosta-1(10),2-diene-4-oic acid (4,9DSHA), 14 9,17-dioxo-1,2,3,4,10,19-hexanorandrostan-5-oic acid (DOHNAA), 15 2-hydroxyhexa-2,4-diene-oic acid (HHD). The compound between brackets is chemically unstable. Hsd 3 $\beta$-hydroxy steroid dehydrogenase, Cyp 125 cytochrome P450 CYP125, KstD 3-ketosteroid dehydrogenase, KSH 3-ketosteroid 9 $\alpha$-hydroxylase, HsaAB 3-hydroxy-9, 10-seconandrost-1,3,5(10)-triene-9,17-dione 4-hydroxylase, $\mathrm{HsaC}$ 3,4-dihydroxy-9,10-secoandrost-1,3,5(10)-triene-9,17dione dioxygenase, HsaD 4,5-9,10-diseco-3-hydroxy-5,9,17trioxoandrosta-1(10),2-diene-4-oic acid hydrolase. The $A, B$, $C$ and $D$ ring of the steroid polycyclic ring structure are indicated in compound 1 (Petrusma 2011)

actinobacteria Rhodococcus equi (Nocardia restrictus) ATCC 14887 (Gibson et al. 1966; Sih et al. 1968), $R$. jostii RHA1 and M. tuberculosis (Van der Geize et al. 2007). Several enzymes have been characterized from these organisms as well as from Rhodococcus erythropolis SQ1 (e.g. Van der Geize et al. 2002), Nocardia corralina (e.g. Itagaki et al. 1990) and $R$. rhodochrous DSM43269 (e.g. Petrusma et al. 2009). Metabolic labelling studies at carbon atom $\mathrm{C} 4$ of cholesterol in $M$. tuberculosis revealed that this carbon atom in the ring structure is converted to $\mathrm{CO}_{2}$, suggesting the generation of energy via the 
tricarboxylic acid (TCA)-cycle (Pandey and Sassetti 2008). Opening of the steroid polycyclic ring structure in these organisms occurs via the so called 9,10-seco pathway. Recently, an alternative 2,3-seco pathway was proposed for aerobic sterol degradation, which does not use oxygenase enzymes for side chain cleavage and for the steroid ring opening event. In the latter case, cleavage of the steroid polycyclic ring structure starts with a hydrolytic attack on the A-ring. The proposed pathway is based on identification of cholesterol degradation intermediates in the gram negative bacterium Sterolibacterium denitrificans (Wang et al. 2013).

In actinobacteria, employing the 9,10-seco pathway, the steroid B-ring is opened by dehydrogenation of the steroid A-ring and by C $9 \alpha$-hydroxylation. The sequence of these reactions is not known. The dehydrogenation step is performed by 3-ketosteroid dehydrogenase enzymes (KSTD) (Itagaki et al. 1990; Knol et al. 2008; Van der Geize et al. 2000), whereas 3-ketosteroid $9 \alpha$-hydroxylase enzymes (KSH) incorporate a hydroxyl moiety at C9 (Van der Geize et al. 2002). The resulting steroid structure, $9 \alpha$-hydroxy1,4-androstadiene-3,17-dione (9OHADD), is chemically unstable and spontaneously hydrolyzes to the phenol 3-hydroxy-9,10-secoandrost-1,3,5(10)-triene9,17-dione (3-HSA). 3-HSA is further degraded by the HsaABCD enzymes. This involves C4-hydroxylation by the flavin-dependent monooxygenase HsaAB (Dresen et al. 2010), cleavage of the A-ring by the extradiol dioxygenase HsaC (Yam et al. 2009) and hydrolysis by HsaD (Lack et al. 2010) yielding 9,17-dioxo1,2,3,4,10,19-hexanorandrostan-5-oic acid (DOHNAA) and 2-hydroxyhexa-2,4-diene-oic acid (HHD). The fate of the DOHNAA product is not entirely clear yet. HHD is further degraded to propionic acid and pyruvate. The two main events in microbial sterol catabolism are $\mathrm{C} 17$-side chain degradation and opening of the polycyclic ring structure. The sequence of these events may differ between organisms. $R$. jostii RHA1 is thought to first attack the sterol side chain followed by opening of the steroid ring structure (Rosłoniec et al. 2009). In M. tuberculosis H37Rv both events may occur simultaneously, since its KSH displays high substrate preference for a CoA thioester intermediate of cholesterol side chain degradation (Capyk et al. 2011). In bile acid transformations with three Rhodococcus strains, 9,10-secosteroid intermediates with partially degraded $\mathrm{C} 17$ side chains were detected, suggesting that $\mathrm{C} 17$-side chain degradation and opening of the steroid polycyclic ring structure occur simultaneously (Costa et al. 2013a, b).

Although microbial steroid hydroxylation is well documented, starting with the discovery of progesterone $11 \alpha$-hydroxylation by a Rhizopus species in 1952 (Peterson and Murray 1952), the responsible enzymes are in most cases not known. Over the years, several cytochrome $\underline{\mathrm{P}} 450$ (CYP) enzymes were found to be involved in steroid hydroxylation (Donova and Egorova 2012 (review); Fernandes et al. 2003 (review); Holland 1999 (review); Rosłoniec et al. 2009). Examples of microbial steroid hydroxylation mediated by $\mathrm{P} 450$ enzymes are $11 \alpha$-hydroxylation by Rhizopus nigricans (Breskvar et al. 1991), 11ßhydroxylation by Curvularia lunata (Suzuki et al. 1993) and $14 \alpha$-hydroxylation by Mucor piriformis (Madyastha and Joseph 1993) and the 26/27-hydroxylases CYP125 of Rhodococcus jostii RHA1 (Rosłoniec et al. 2009) and CYP142 of Mycobacterium tuberculosis (Driscoll et al. 2010).

Various steroid hydroxylases play a role in microbial steroid degradation, resulting in hydroxylation of steroids at virtually every carbon atom (Fernandes et al. 2003; Mahato and Garai 1997; Donova 2007; Donova and Egorova 2012 (reviews)). Pharmaceutical steroids have a wide range of applications and hydroxylated steroids, often displaying high bioactivity, are of large industrial and medical relevance. The pharmaceutically most interesting sites for hydroxylation are at carbon atoms C-7 $\alpha, \mathrm{C}-9 \alpha, \mathrm{C}-11 \alpha, \mathrm{C}-11 \beta$, C-16 $\alpha$, C-17 $\alpha$ (Donova and Egorova 2012 (review)). Steroid C9-hydroxylation and C11-hydroxylation reactions for instance are important for the production of corticosteroids (Kano et al. 1979; Megges et al. 1990).

Steroid $\mathrm{C} 9 \alpha$-hydroxylation is catalyzed by the 3-ketosteroid 9 $\alpha$-hydroxylase (KSH) enzyme. KSH is a key-enzyme in bacterial steroid degradation and initiates opening of the steroid polycyclic ring structure. The two-component Rieske-type non-heme monooxygenase $\mathrm{KSH}$ consists of the terminal oxygenase KshA and the ferredoxin reductase KshB. Deletion of KshA activity in sterol degrading bacteria results in blockage of steroid ring opening and is used to produce valuable C19-steroids, such as 4-androstene-3,17-dione (AD) and 1,4-androstadiene-3,17dione (ADD), core metabolites in the sterol degradation pathway (Andor et al. 2006; Van der Geize et al. 
2002; Wilbrink et al. 2011). These steroids can be used as precursors for virtually all pharmaceutically interesting steroids (Wang et al. 2011 (review)). Interestingly, it has been reported that KSH is essential for the pathogenicity of M. tuberculosis (Hu et al. 2010). Insight into this important enzyme can provide new leads for the development of inhibitors to be used as drugs against this notorious pathogen.

\section{3-Ketosteroid $9 \alpha$ hydroxylase, a multimeric two component Rieske type non-heme oxygenase}

$\mathrm{KSH}$ is a key enzyme in bacterial sterol degradation, initiating the opening of the steroid polycyclic ring structure by C $9 \alpha$-hydroxylation (Gibson et al. 1966; Van der Geize et al. 2002). Characterization of enzymes involved in C $9 \alpha$-hydroxylation of steroids started with a partly purified NADH dependent three component enzyme system of Nocardia sp. M117 (Strijewski 1982). The enzymes were identified as a flavoprotein reductase and two iron-sulphur proteins. Genes encoding a two component KSH enzyme system were first identified in $R$. erythropolis SQ1 (Van der Geize et al. 2002). UV-mutagenesis of $R$. erythropolis SQ1 and screening and characterization of mutants impaired in growth on AD but still able to grow on $9 \alpha$-hydroxyAD (9OHAD) revealed that the $k s h A$ and $k s h B$ genes are involved in steroid $C 9 \alpha-$ hydroxylation. Unmarked gene deletion in $R$. erythropolis SQ1 (van der Geize et al. 2001) of either $k s h A$ or $k s h B$ both resulted in mutant strains incapable of growth on AD. Based on its amino acid sequence, KSH was identified as a Rieske non-heme monooxygenase enzyme system. Monooxygenases are also called mixed-function oxygenases because they incorporate one oxygen atom into the substrate and one atom is reduced to $\mathrm{H}_{2} \mathrm{O}$ (Harayama et al. 1992; Mason and Cammack 1992 (reviews)).

Rieske non-heme oxygenases (ROs) constitute a distinct class of enzymes. They are found in a wide range of organisms but occur most abundantly in bacteria. The members of this enzyme family are involved in catabolism of a wide range of substrates, including many aromatic and toxic compounds (Chakraborty et al. 2012). Therefore, these enzymes are of great environmental and industrial importance. ROs are multicomponent redox enzyme systems, consisting of an oxygenase and one or two reducing enzymes. The oxygenase component, which performs the substrate hydroxylation, is an iron-sulphur protein and contains a non-heme iron situated at the active site. KSH enzymes employ an electron transport chain that starts with the oxidation of NADH. The electrons are transferred to the flavin co-factor (FAD) of the ferredoxin reductase component $\mathrm{KshB}$ and then transported to the plant type iron-sulphur cluster of KshB. The Rieske iron-sulphur cluster of the KshA oxygenase component subsequently accepts the electrons from KshB. The electrons end up at the nonheme iron situated in the active site of KshA. The mononuclear iron is the site where $\mathrm{O}_{2}$ is bound and activated and the substrate is hydroxylated (Batie et al. 1991; Mason and Cammack 1992 (review)) (Fig. 2). The crystal structure of KshA of M. tuberculosis revealed that the Rieske iron-sulphur cluster and the non-heme $\mathrm{Fe}^{2+}$ catalytic centre are located relatively far away from each other. Crystal structures from other ROs confirm that this is characteristic for this type of protein. However, the typical head-to-tail trimer arrangement positions the Rieske $\mathrm{Fe}_{2} \mathrm{~S}_{2}$ in close proximity to the non-heme $\mathrm{Fe}^{2+}$ of the neighbouring KshA subunit, enabling transport of electrons between KshA subunits (Fig. 3). A conserved aspartic acid, Asp178 of KshA of M. tuberculosis (Capyk et al. 2009), plays a key role in this arrangement by formation of hydrogen bonds, thereby facilitating electron transfer between adjacent subunits (Parales et al. 1999). This aspartate has also been implicated in catalysis (Beharry et al. 2003; Pinto et al. 2006; Tarasev et al. 2006). A common feature of non-heme iron enzymes is the 2-His-1-carboxylate facial triad motif binding the non-heme iron in the core of the active site. The iron is thus coordinated in the protein by three endogenous ligands leaving it largely exposed and creating a very reactive active site (Fig. 2). Up to three exogenous ligands can be bound to the metal centre (Bruijnincx et al. 2008; Hegg and Que 1997 (reviews)). The metal centre of KshA is coordinated at the core of the active site by two histidines (His181 and His 186) and an aspartate (Asp304) residue (Capyk et al. 2009). As in several Rieske oxygenases, the iron is bidentate bound to the carboxyl group of the aspartate leaving two sites available for exogenous ligands. This metal centre is more labile compared to the covalently bound heme-iron. However, non-heme iron is a highly catalytic platform able to bind $\mathrm{O}_{2}$ and 


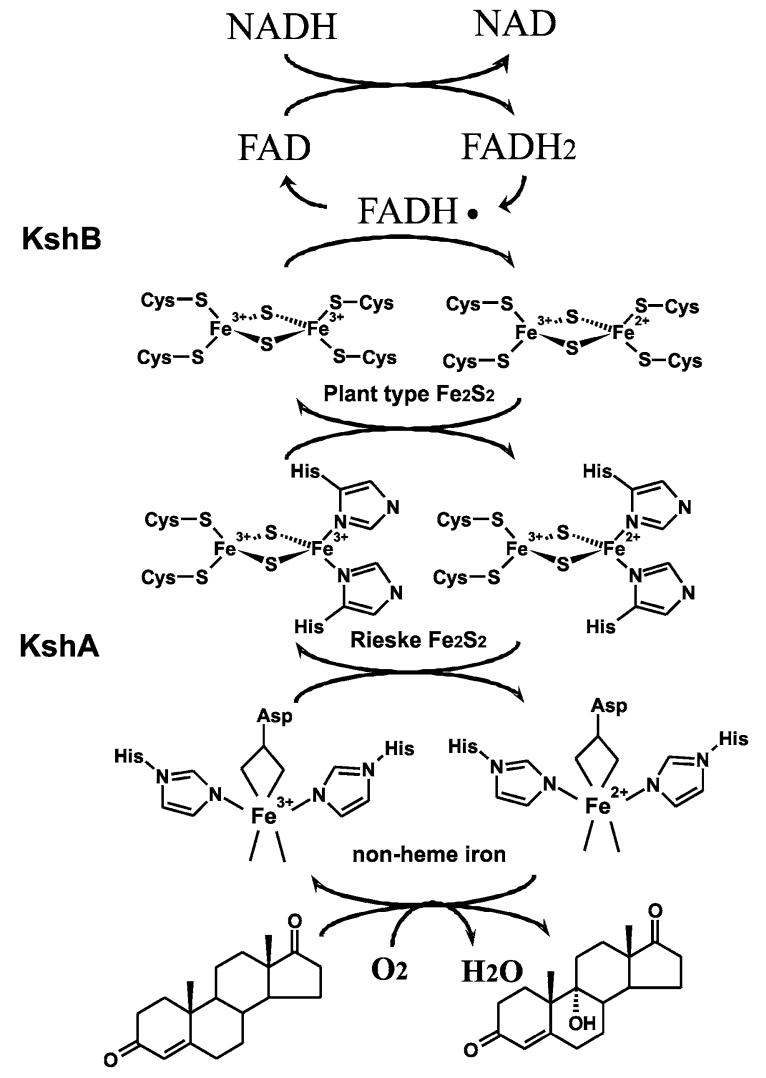

Fig. 2 Electron transport chain reactions and substrate hydroxylation catalyzed by KSH. Arrows indicate the flow of electrons, starting from the electron donor NADH to the flavin co-factor of $\mathrm{KshB}, \mathrm{FAD}$, and via the plant type iron-sulphur cluster, coordinated by four cysteines, of KshB to the Rieske ironsulphur cluster, coordinated by two cysteines and two histidines, of KshA ending up at the non-heme iron at the core of the catalytic domain. Non-heme iron is coordinated by an aspartate and two histidine residues leaving two binding sites open. Here $\mathrm{O}_{2}$ can be bound and one $\mathrm{O}$-atom is used for the hydroxylation of the steroid substrate while the other $\mathrm{O}$-atom is reduced to $\mathrm{H}_{2} \mathrm{O}$ (Petrusma 2011)

steroid substrate simultaneously in different orientations.

Rieske oxygenases are known to catalyze a range of chemical reactions such as dioxygenation and monooxygenation. Several ROs are known to perform both reactions, e.g. carbazole 1,9a-dioxygenase (Nojiri et al. 1999). Furthermore, ROs are also known to catalyze reactions like desaturation (e.g. Torok et al. 1995), O-dealkylation (Resnick and Gibson 1993), sulfoxidation (Boyd et al. 2004; Lee et al. 1995), desulphonative dioxygenation (Locher et al. 1991) and oxidative carbocyclization (Sydor et al. 2011). This range of reactions performed by ROs demonstrates the

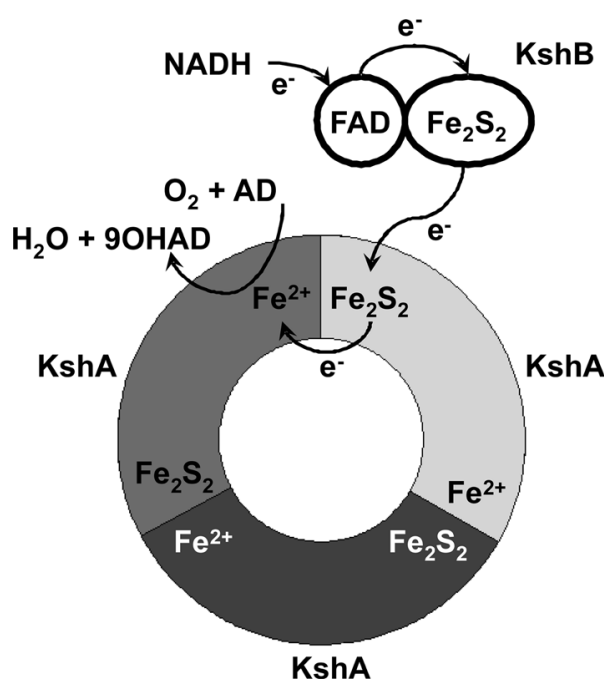

Fig. 3 Schematic representation of the typical head-to-tail trimer arrangement of KshA enzymes (adapted from Capyk et al. 2009). Three KshA units are shown in light grey, dark grey and black, forming a circle. Also the FAD co-factor and the iron-sulphur cluster of KshB are depicted. Electrons (e-) are transferred from the iron-sulphur cluster of KshA to the nonheme $\mathrm{Fe} 2+$ of the neighbouring KshA unit. AD and 9OHAD indicate 4-androstene-3,17-dione and 9 $\alpha$-hydroxy-4-androstene-3,17-dione, respectively (Petrusma 2011)

large catalytic capacity of the non-heme iron active site. The reaction mechanism of $\mathrm{KSH}$ is unknown and although many studies provide insight into the nature of catalysis of ROs, the precise catalytic mechanism of ROs is still unclear. However, some common mechanistic features are apparent for ROs. The first step in catalysis is binding of the substrate in the active site and reduction of the non-heme iron $\left(\mathrm{Fe}^{2+}\right)$. Furthermore, the Rieske iron-sulphur cluster also needs to be in a reduced state. Only when the oxygenase is in this state, $\mathrm{O}_{2}$ can bind to the metal centre. This arrangement prevents uncoupling because all components of the reaction are necessary for activation of $\mathrm{O}_{2}$ (Barry and Challis 2013; Neidig and Solomon 2005 (reviews)). Crystal structures of naphthalene dioxygenase provided evidence for side-on $\left(\eta^{2}\right)$ binding of $\mathrm{O}_{2}$ to the metal centre, meaning that both $\mathrm{O}$-atoms are bound to the $\mathrm{Fe}^{2+}$. This type of $\mathrm{O}_{2}$ binding may explain the high regiospecificity observed for many ROs. This contrasts with CYP enzymes, which bind $\mathrm{O}_{2}$ in an end-on fashion, meaning that only one oxygen atom is bound to the heme iron (Karlsson et al. 2003). The versatility and high regiospecificity of ROs makes the enzymes very interesting for industrial applications. 
The oxygenase component of ROs is either an $\alpha$ subunit alone, e.g. KshA, the oxygenase component of $\mathrm{KSH}$, or contains both an $\alpha$-subunit and a $\beta$-subunit (Mason and Cammack 1992 (review)). The $\beta$-subunit is smaller and its function has not been elucidated. Several studies indicate a role for the $\beta$-subunit in substrate hydroxylation activity (e.g. Jiang et al. 1999). However, other studies indicate that the $\beta$ subunit has a structural role and is not involved in substrate specificity or selectivity (e.g. Friemann et al. 2005). 3D structures are available for a range of Rieske non-heme oxygenases. All enzymes are organized in either an $\alpha 3$ trimer, e.g. KshA (Capyk et al. 2009) (Fig. 3) or an $\alpha 3 \beta 3$ hexamer, e.g. nitrobenzene dioxygenase (Friemann et al. 2005). An exception is the phthalate dioxygenase of Burkholderia cepacia (DB01) which is organized as an $\alpha 3 \alpha 3$ stacked hexamer (Tarasev et al. 2007). The $\alpha$-subunit, e.g. KshA, contains a Rieske domain, coordinating the Rieske $\mathrm{Fe}_{2} \mathrm{~S}_{2}$ cluster, and a catalytic domain with the typical helix-Grip fold, which is part of the StAR (steroidogenic acute regulatory protein) related lipid transfer (START) domain superfamily. The catalytic domain is composed of a $\beta$-sheet flanked by $\alpha$-helices (Iyer et al. 2001). The oxygenase component of ROs is reduced by a ferredoxin reductase, e.g. KshB in KSH. In three component systems the electrons are shuttled between the oxygenase and the reductase by a small ferredoxin (Mason and Cammack 1992(review)). This ferredoxin has not been detected for KSH.

Amino acid sequence analysis of KshA (oxygenase) reveals a Rieske iron-sulphur binding motif and a non-heme $\mathrm{Fe}^{2+}$ binding motif, and KshB (reductase) has a flavin, NADH and plant type iron-sulphur binding motifs (Van der Geize et al. 2002) (Fig. 4). KSH was thus classified als a class IA RO enzyme system according to the RO classification system proposed by Batie et al. (1991) (Table 1). This classification is based on the diversity of electron transport components in the oxygenases. The terminal oxygenase is dependent on a ferredoxin reductase for reduction. Several RO systems need a third protein, a small ferredoxin that shuttles electrons between the ferredoxin reductase and the terminal oxygenase. Location, number and nature of the iron-sulphur clusters are used as characteristics to group oxygenases. The Batie classification system consists of three main classes, of which class I and class II are subdivided in group A and B. Class I oxygenases consist of two components, a terminal oxygenase and a ferredoxin reductase. Class IA ferredoxin reductases have N-terminal FMN as co-factor while FAD is cofactor in class IB, located between the iron-sulphur binding domain and the NAD binding domain (Correll et al. 1992). Class II and III oxygenases employ ferredoxin as a third component. In class II systems the ferredoxin reductase contains FAD and the ferredoxin an iron-sulphur cluster. Class II is subdivided in group $\mathrm{A}$ and $\mathrm{B}$ on the basis of the nature of the iron-sulphur cluster of the ferredoxin. In class III the ferredoxin reductase contains both a FAD and an iron-sulphur cluster. The reductase component of class III shows strong similarity to the reductase of class IB. However, in the class III systems a ferredoxin, containing an iron-sulphur cluster is required for electron transfer between the reductase and the oxygenase (Batie et al. 1991).

The Batie classification system for ROs has been broadly applied. The biochemical classification has a strong evolutionary basis (Harayama et al. 1992 (review); Nakatsu et al. 1995; Neidle et al. 1991). However with the identification of a growing number of ROs, more and more enzymes are found to have characteristics that do not fit the Batie classification system. KSH was also initially classified as a Class IA RO based on amino acid sequence analysis. However, biochemical characterization of KshB showed the presence of FAD as co-factor indicative for a class IB oxygenase (Capyk et al. 2009; Petrusma et al. 2009).

A new classification system was introduced by Nam et al. (2001) based on the amino acid sequence of the oxygenase components (the alpha subunits). Evolutionary relationships among oxygenases do not appear to be related to substrate specificity, since oxygenases sharing similar substrate ranges can be classified in different groups, based on amino acid sequence similarity. The Nam-system consists of four groups. KSH was classified as a group I RO in this classification system. Group I contains a broad range of mono- and dioxygenases with low amino acid sequence similarity and various protein sizes. The oxygenases are $\alpha$-monomers. Sequence alignments did not allow elucidation of the specific domains necessary for monooxygenation or dioxygenation specificity. Characteristic for group I oxygenases are 16 or 18 residues between the first histidine residue and the second cysteine residue of the Rieske ironsulphur binding domain. In contrast, oxygenases of 


\begin{tabular}{|c|c|c|c|}
\hline & \multicolumn{2}{|r|}{ Rieske $\mathrm{Fe}_{2} \mathrm{~S}_{2}$ binding motif } \\
\hline & & & * * $\quad$ * \\
\hline KshA1 & (R. ery) & 77 & daycrhmggnlahgtvkgdsiacpfhdwr \\
\hline ksha2 & (R. ery) & 72 & daycrhmggdlsqgtvkgdsvacp fhdwr \\
\hline KshA & (Ro04538) & 65 & daycrhmggdlsqgtvkgdsvacp fhdwr \\
\hline KshA2 & (Ro02490) & 77 & daycrhmggnlahgtvkgdsiacp fhdwr \\
\hline KshA3 & (Ro05811) & 79 & dgycrhmggdlsqgtvkgdevacp fhdwr \\
\hline KshA4 & (Ro09003) & 65 & nafcphmggnladgtvkgdtiacp fhdwr \\
\hline KshA & (MSMEG 5925) & 62 & daycrhmggdlskgtvkgdevacp fhdwr \\
\hline KshA & (RV352它) & 64 & dgycrhmggdlsegtvkgdevacp fhdwr \\
\hline KshA1 & (R. rho) & 65 & daycrhmggdlsqgtvkgdeiacp fhdwr \\
\hline KshA2 & (R. rho) & 64 & daycrhmggnlaqgsvkgntiacp fhdwr \\
\hline KshA3 & (R. rho) & 58 & daycrhmggdlsqgeikgdsvacp fhdwr \\
\hline KshA4 & (R. rho) & 64 & dafcrhmggnlargeikgdtiacp fhdwr \\
\hline KshA5 & (R. rho) & 70 & daycrhmggdlsrgevkgdsiacpfhdwr \\
\hline
\end{tabular}

\begin{tabular}{|c|c|c|c|}
\hline & & $\begin{array}{l}\text { flavin binding motif } \\
\star \star \star \star \star\end{array}$ & NADH binding motif \\
\hline KshB & (R. ery) & 61 svarcyslass & 124 lfgagsgitpvi \\
\hline KshB3 & (Ro05833) & 61 svarcyslass & 124 lfaagsgitpvm \\
\hline KshB4 & (Ro09002) & 60 gtarcyslsss & 124 lvaggsgitpvi \\
\hline KshB & (RV3571) & 69 svarcyslcss & 132 llaagsgitpims \\
\hline KshB & (R. rho) & 61 svarcyslass & 123 lfgagsgitpvis \\
\hline
\end{tabular}

Fig. 4 Co-factor binding amino acid sequence motifs in KshA and KshB of $R$ erythropolis SQ1 (R. ery) (KshA1, KshB (Van der Geize et al. 2002); KshA2 (Van der Geize et al. 2008), $R$. jostii RHA1 (Ro gene numbering) (Mathieu et al. 2010; Van der Geize et al. 2007). M. smegmatis $\mathrm{mc}^{2} 155$ (MSMEG_5925) non-heme iron binding motif

184 eivdnvvdmahffyvhysf

177 eivdnvvdmahffyvhfsf

177 eivdnvvdmahffyvhfsf

184 eivdnvvdmahfyyvhfsf

185 evvdnivdmahffyvhys $f$

172 eivdnvvdmahffyvhygm

169 eiidnvtdmahffyihfgl

171 diidnvtdmahffyihfgl

171 eiidnivdmahffyvhys $f$

171 eivdnvvdmahffyvhygm

165 eiidnvvdmahffyihyaf

171 eivdnvvdmahffyvhfqm

177 eivdnvvdmahffyihys

Table 1 Classification system of Rieske non-heme oxygenases according to Batie et al. (1991)

\begin{tabular}{lllll}
\hline Class & $\begin{array}{l}\text { Number } \\
\text { of components }\end{array}$ & $\begin{array}{l}\text { Cofactor of the } \\
\text { ferredoxin reductase }\end{array}$ & $\begin{array}{l}\text { Iron-sulphur cluster } \\
\text { in ferredoxin }\end{array}$ & $\begin{array}{l}\text { Iron-sulphur cluster } \\
\text { in oxygenase }\end{array}$ \\
\hline IA & 2 & FMN $\left[\mathrm{Fe}_{2} \mathrm{~S}_{2} \mathrm{Cys}_{4}\right]$ & - & {$\left[\mathrm{Fe}_{2} \mathrm{~S}_{2} \mathrm{Cys}_{2} \mathrm{His}_{2}\right]$ non-heme $\mathrm{Fe}^{2+}$} \\
IB & 2 & FAD $\left[\mathrm{Fe}_{2} \mathrm{~S}_{2} \mathrm{Cys}_{4}\right]$ & - & {$\left[\mathrm{Fe}_{2} \mathrm{~S}_{2} \mathrm{Cys}_{2} \mathrm{His}_{2}\right]$ non-heme $\mathrm{Fe}^{2+}$} \\
IIA & 3 & FAD & {$\left[\mathrm{Fe}_{2} \mathrm{~S}_{2} \mathrm{Cys}_{4}\right]$} & {$\left[\mathrm{Fe}_{2} \mathrm{~S}_{2} \mathrm{Cys}_{2} \mathrm{His}_{2}\right]$ non-heme $\mathrm{Fe}^{2+}$} \\
IIB & 3 & FAD & {$\left[\mathrm{Fe}_{2} \mathrm{~S}_{2} \mathrm{Cys}_{2} \mathrm{His}_{2}\right]$} & {$\left[\mathrm{Fe}_{2} \mathrm{~S}_{2} \mathrm{Cys}_{2} \mathrm{His}_{2}\right]$ non-heme $\mathrm{Fe}^{2+}$} \\
III & 3 & FAD $\left[\mathrm{Fe}_{2} \mathrm{~S}_{2} \mathrm{Cys}_{4}\right]$ & {$\left[\mathrm{Fe}_{2} \mathrm{~S}_{2} \mathrm{Cys}_{2} \mathrm{His}_{2}\right]$} & {$\left[\mathrm{Fe}_{2} \mathrm{~S}_{2} \mathrm{Cys}_{2} \mathrm{His}_{2}\right]$ non-heme $\mathrm{Fe}^{2+}$} \\
\hline
\end{tabular}

group II, III and IV all have 17 residues separating the first histidine and second cysteine. The two histidines binding the non-heme $\mathrm{Fe}^{2+}$ are separated by 3 or 4 amino acids in class I, 4 amino acids in class II and III and 4 or 5 amino acids in class IV. The system proposed by Nam et al. (2001) also allows classification according to the electron transport chain. Group II oxygenases are composed of two components, groups III and IV are made up of three components and group I has either 2 or 3 components. The Batie et al. (1991) and Nam et al. (2001) classification systems are based on the composition of the electron transport chain of oxygenases and on the amino acid sequences of the oxygenases, respectively. Together, they complement each other and give more insight into similarities and differences of ROs. In 2008, another classification system was introduced by Kweon et al. (2008) that incorporated both information about the composition of the electron transport chain as well as the oxygenase component. This classification system is based on information of all components of ROs but is also reliable in classifying incomplete oxygenases systems. Thus, this classification system can predict the presence of unknown electron transport components. The classification consists of five types of oxygenases. KshA was classified as a Type I oxygenase in the Kweon et al. (2008) classification system. A modification of the Kweon classification was suggested by 
Chakraborty et al. 2012. This modification includes combinations of electron transport chain components based on evolutionary relationships between the different RO components as well as functional properties of the ROs. Oxygenase components of ROs share structural similarities but are very diverse in amino acid sequence. A phylogenetic study, combining structural information and amino acid sequences of the oxygenase components of ROs, suggest that classification of ROs in such broad groups is not feasible (Capyk and Eltis 2012). The RO phylogenetic tree shows two distinct groups but within these groups specific clans cannot be identified. One exception is a large clan in group II, also containing KshA of $M$. tuberculosis H37Rv. Interestingly, KshA is clustered with the eukaryotic Neverland enzyme, a cholesterol 7,8-dehydrogenase. The authors propose a new classification system which is constituted of smaller groups, with functional similarities between the members of such a group. This system would be similar to the naming system of P450s. Since this new classification system is focussed on the oxygenase component of ROs, the system introduced by Batie et al. 1991 would complement the new system because it also includes the other components of the electron transport chain of ROs (Capyk and Eltis 2012).

After the identification of $\mathrm{KSH}$ in $R$. erythropolis SQ1 (van der Geize et al. 2001) a kshA gene was also identified in Mycobacterium smegmatis mc2 155. E. coli cells expressing this KshA displayed $C 9 \alpha-$ hydroxylating activity on AD and progesterone. Upon purification of the enzyme the hydroxylating activity was greatly reduced (Andor et al. 2006; Arnell et al. 2007). $k s h A$ and $k s h B$ of $R$. rhodochrous DSM43269 were co-expressed in $E$. coli and whole cell bioconversion of AD to 9OHAD was observed, with a yield of $>60 \%$ after 48 h (Petrusma et al. 2009). KshA and KshB of both $R$. rhodochrous DSM43269 (Petrusma et al. 2009) and M. tuberculosis (Capyk et al. 2009) has been purified in active forms and were dependent on NADH as electron donor for activity. KshA of $M$. tuberculosis was purified anaerobically (Capyk et al. 2009) while KshA of $R$. rhodochrous DSM43269 was purified aerobically but only remained in an active form when purified together with KshB, resulting in an active KSH enzyme (Petrusma et al. 2009). The 3D structure of KshA of M. tuberculosis has been elucidated (Capyk et al. 2009). It contains a Rieske iron-sulphur cluster and a divalent non-heme iron located in the core of the active site. KshA subunits are organized in trimers, forming a typical $\alpha 3$-fold as mentioned above (Fig. 3). The catalytic domain of KshA is organized in the typical Helix-Grip fold (see above) but differs from other known $\alpha 3$-ROs in the shape of the substrate-binding pocket and position of active site channel, equipped for steroid substrates. $\mathrm{KshA}$ has distinct C-terminal features involved in formation of the $\alpha$-trimer. It was suggested that KshA is an archetypical RO because the structure shows a minimal catalytic domain compared to other available RO structures (Capyk et al. 2009) (Fig. 5). In vitro bioassays with KSHs of M. tuberculosis H37Rv and $R$. rhodochrous DSM43269 showed that the enzymes can use 3-ketosteroids as substrates. They can tolerate different configurations of the A-ring and some enzymes display hydroxylation activity on steroids with longer $\mathrm{C} 17$ side chains. As mentioned above, KSH of M. tuberculosis shows high preference for the CoA thioester intermediate of cholesterol side chain degradation compared to the tested C17-ketosteroids. These studies indicate that $\mathrm{KSH}$ activity can occur at different stages of the sterol degradation pathway (Capyk et al. 2011; Petrusma et al. 2011).

\section{Physiological roles of KSH enzymes}

Sterols and steroids can be degraded by a range of microorganisms, many of which are actinobacteria, e.g. Rhodococcus and Mycobacterium species, and serve as carbon- and energy sources for growth (Donova 2007; Fernandes et al. 2003; Malaviya and Gomes 2008; Van der Geize and Dijkhuizen 2004 (reviews)). KSH is a key enzyme in bacterial sterol catabolic pathways. The essential role of $\mathrm{KSH}$ in opening of the steroids polycyclic ring structure was demonstrated for the first time in R. erythropolis SQ1. The $k s h A$ and $k s h B$ gene deletion mutants were able to grow on 9-OHAD but not on AD. This strain, however, was still able to grow on phytosterols, indicating the presence of additional KshA enzymes or an alternative degradation pathway (Van der Geize et al. 2002). A kshA disruption mutant of Mycobacterium smegmatis mc2 155 incubated with sitosterol accumulated AD and ADD (Andor et al. 2006). $R$. rhodochrous DSM43269 expresses 5 KshA homologs. A $k s h A$ null mutant was constructed by gene deletion mutagenesis (strain RG32) to fully block opening of 
Fig. 5 Crystal structure of KshA of M. tuberculosis H37Rv (a), with non-heme iron coordinating residues in orange (His181, His186 and Asp304) and putative substrate interacting residues in blue (Val176, Gln204, Tyr232, Met238, Asn240, Asn257, Phe301, Trp308) (adapted from Capyk et al. 2009, PDB: 2ZYL). Stereo image of an ADD docking experiment indicating substrate interacting residues of KshA (b) (This figure was originally published in Journal of Biological Chemistry. Capyk et al. (2009) 284:9937-9946 (c) the American Society for Biochemistry and Molecular Biology.)

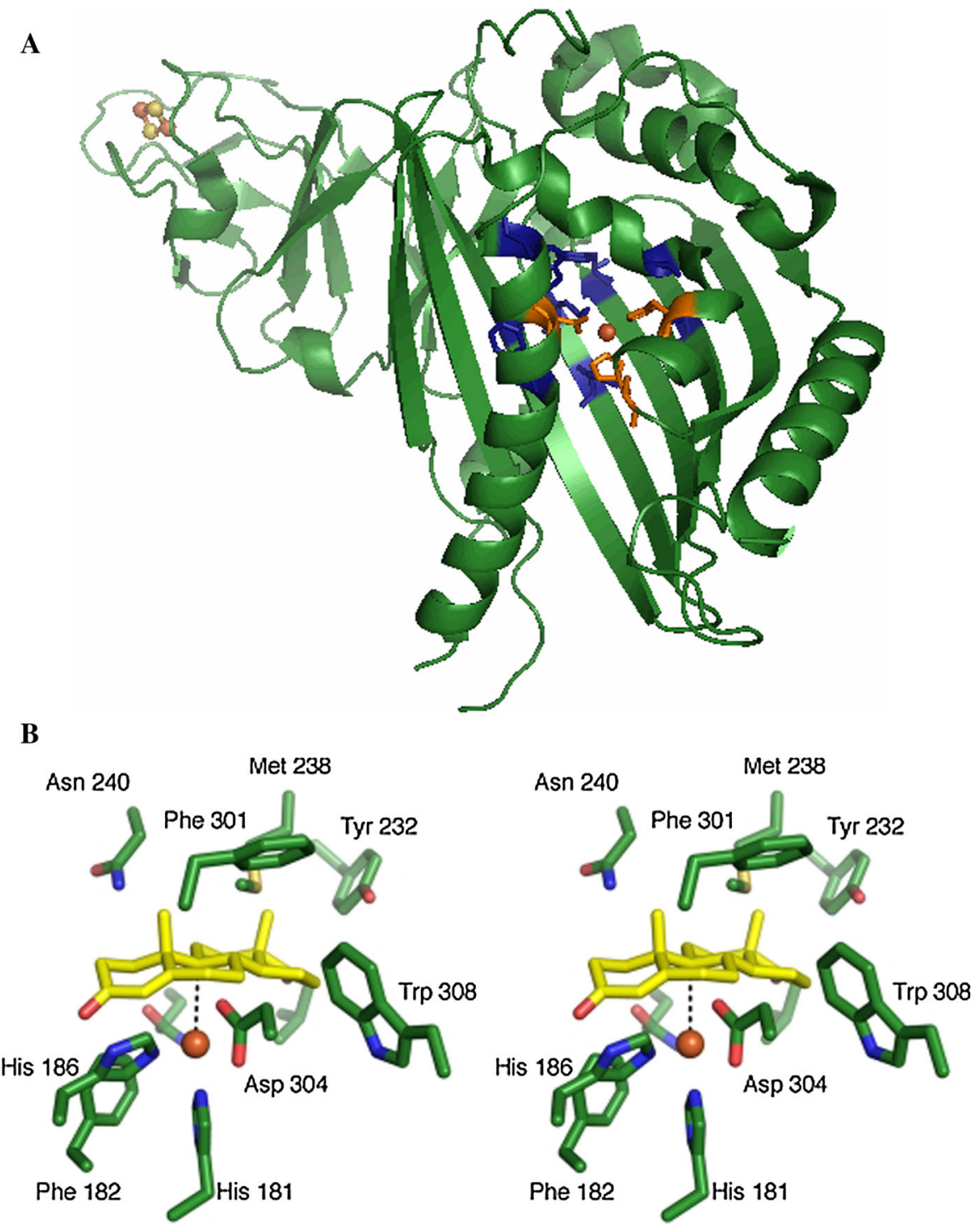

the steroids polycyclic ring structure of cholesterol and $\beta$-sitosterol; this resulted in accumulation of ADD and 3-oxo-23,24-bisnorchola-1,4-dien-22-oic acid (1,4-BNC) (Wilbrink et al. 2011). These mutants still degraded the sterol side chain, thus demonstrating the potential of mutant bacterial strains to convert relatively cheap sterol substrates into valuable steroids.

Rhodococci were found to have relatively large genomes, displaying strong gene diversity. Multiple copies are present for many genes, encoding (iso)enzymes for specific steps in catabolic pathways. Such a multiplicity was also observed for $k s h A$. The genome of $R$. erythropolis SQ1 harbours at least $3 k s h A$ genes
(Van der Geize et al. 2008). $R$. jostii RHA1 encodes four sterol catabolic gene clusters, each of which contains a $k s h A$ gene, namely ro04538 ( $k s h A)$, ro02490 (kshA2), ro05811 (kshA3) and ro09003 ( $k s h A 4)$ (Mathieu et al. 2010; McLeod et al. 2006; Van der Geize et al. 2007). A total of five $k s h A$ homologues were identified in $R$. rhodochrous DSM43269 (Wilbrink et al. 2011) and Mycobacterium sp. VKM Ac-1817D (Bragin et al. 2013), and seven $k s h A$ homologues are present in the genome of the pathogenic bacterium $R$. equi. Multiplicity for $k s h B$ is not as excessive as for $k s h A$. Apparently, KshB is able to serve as a reductase for several KshA enzymes. In 
vitro bioassays of the five KshA homologues of $R$. rhodochrous DSM43269 also shows that all KshAs are able to use the same KshB as reductase (Petrusma et al. 2011). Little is known about the physiological roles of the different KshA homologues. In vivo, each of these KshA homologues may have a specific role in the host bacterium, degrading certain sterol/steroid substrates. Sterol catabolic gene cluster 1 of $R$. jostii RHA1 was designated as a cholesterol degradation gene cluster. Most genes within this cluster are upregulated during growth on cholesterol, including $k s h A$ (ro04538). The study showed that several pathogenic bacteria also harbour a cholesterol degradation cluster and in Mycobacterium bovis bacillus Calmette-Guérin kshA was found to be upregulated during growth on cholesterol. A cholesterol catabolic gene cluster was also identified in M. tuberculosis (Van der Geize et al. 2007).

$R$. jostii RHA1 gene expression has been analyzed during growth on 7-ketocholesterol (7-KC) and revealed that a set of genes, among which $k s h A 3$, was specifically upregulated during growth on 7-KC compared to cholesterol. However, many genes were upregulated on both sterols suggesting a common degradation pathway (Mathieu et al. 2010). Promoter activity experiments with $k s h A 2$ of $R$. erythropolis SQ1 showed that $k s h A 2$ is highly induced by $9 \alpha-$ hydroxy-4-androstene-3,17-dione (9-OHAD), a KSH product. It was speculated that this $k s h A$ homologue prevents accumulation of ADD to toxic levels during sterol catabolism (Van der Geize et al. 2008). Molecular studies with the five KshA homologues of $R$. rhodochrous DSM43269 indicated that these isoenzymes are involved in degradation of specific types of sterols and/or at different levels in the degradation cascade. KshA1 of $R$. rhodochrous DSM43269 appears to be dedicated to cholate catabolism. Expression of $k s h A l$ was induced during growth on cholic acid. Furthermore, the growth of a $R$. rhodochrous DSM43269 kshA null mutant, unable to grow on cholate, was restored to wild type level by complementation by KshA1, and the KshA1 enzyme also showed substrate preference for an intermediate in cholate catabolism (Petrusma et al. 2011). KshA1 of $R$. rhodochrous and KshA3 of $R$. jostii RHA1 are clustered in the same group in a KshA phylogenetic tree (Fig. 6). Phylogenetic analysis showed clustering of KshA enzymes in different groups. It was hypothesized that the members of such a group are functionally related, involved in catabolism of specific steroids (Petrusma et al. 2011). The gene cluster of $R$. jostii RHA1 harboring kshA3 (ro05811) was found to be essential for cholate catabolism. Many genes in the cluster, among which $k s h A 3$, were found to be highly upregulated during growth on cholate compared to cholesterol. Several bacteria harbouring orthologous gene clusters in their genomes were also found to be able to grow on cholate while all tested bacteria lacking such a gene cluster were unable to grow on this bile acid. A $k s h A 3$ deletion mutant confirmed that the KshA3 enzyme is essential for growth on cholate (Mohn et al. 2012).

\section{KSH is essential for the pathogenicity of M. tuberculosis}

M. tuberculosis has a much smaller genome than most Rhodococcus species characterized and does not display the high gene multiplicity characteristic for most rhodococci. Indeed, the genome of $M$. tuberculosis harbours one $k s h A$ gene and one $k s h B$ gene. $M$. tuberculosis is one of the most notorious human pathogenic bacteria. Each year approximately 1.5 million deaths are caused by the disease tuberculosis and millions of people are infected (www.who.int/tb/ country/en/index.html). M. tuberculosis is such a successful pathogen because it is able to survive for long periods of time (chronic) within macrophages in the lungs (Meena and Rajni 2010 (review)). In recent years it was found that cholesterol catabolism plays an important role in the survival of M. tuberculosis in macrophages (Rengarajan et al. 2005; Sassetti and Rubin 2003; Schnappinger et al. 2003; Van der Geize et al. 2007). The precise role of cholesterol degradation in M. tuberculosis however is not clear. Several enzymes were found to be essential for survival of $M$. tuberculosis during infection (see below), but cholesterol does not serve as the sole energy source during infection (Yang et al. 2011). It has been suggested that steroid intermediates of the bacterial cholesterol degradation pathway modulate the immune response of the human host (Klink et al. 2013; Yam et al. 2011 (review)).

Several studies have shown that cholesterol catabolism in M. tuberculosis is essential in the chronic phase of infection and in IFN- $\gamma$-activated macrophages but not during the onset of infection or in 
Fig. 6 Phylogenetic tree of bacterial KshA enzymes (Petrusma et al. 2011)

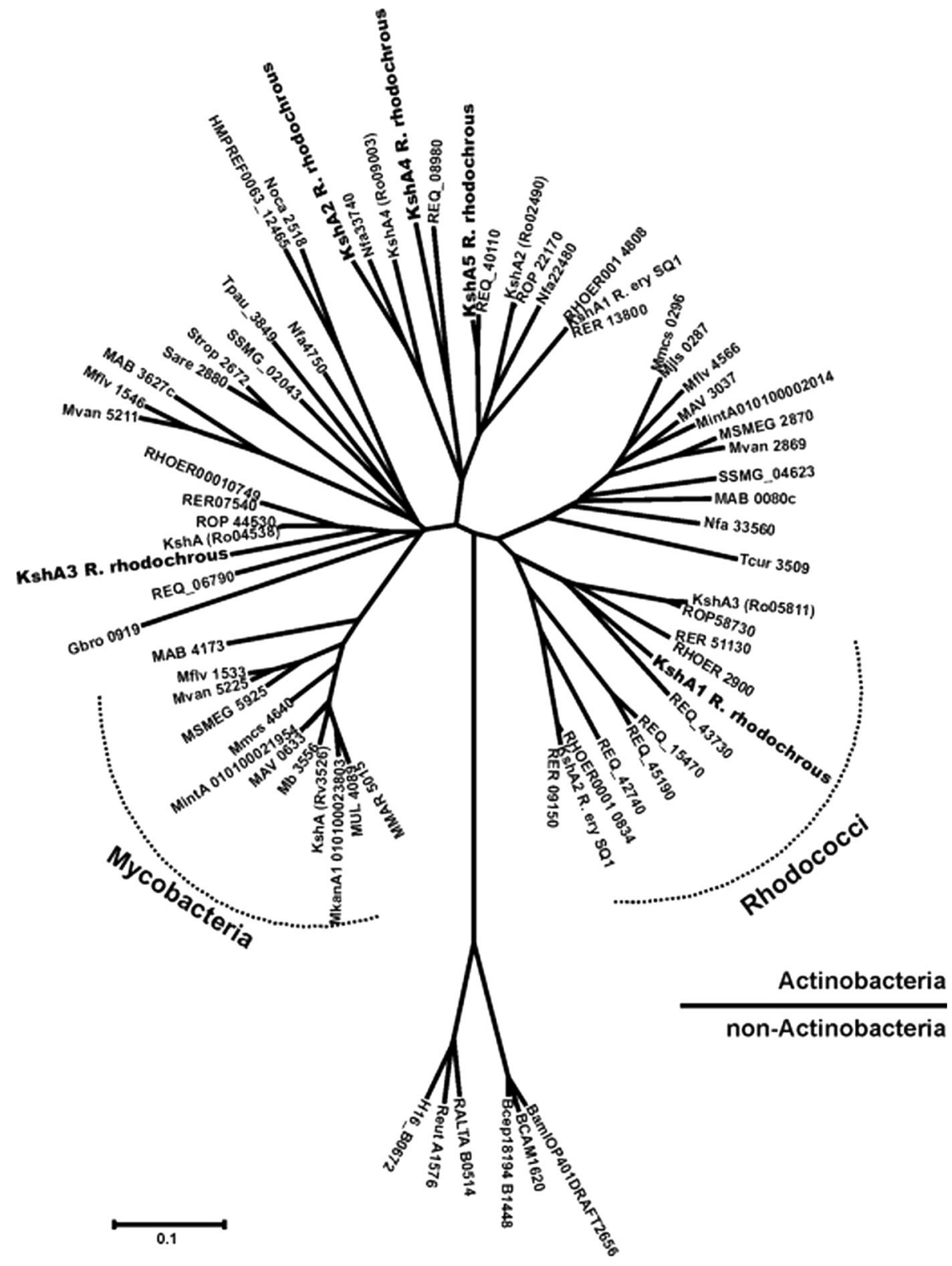

resting macrophages (Nesbitt et al. 2010; Pandey and Sassetti 2008). However, transposon mutagenesis studies (Rengarajan et al. 2005; Sassetti and Rubin 2003) and targeted gene inactivation studies of genes coding for enzymes involved in steroid ring degradation (Hu et al. 2010; Yam et al. 2009), indicated that sterol catabolism is essential in the early stages of infection as well as in the chronic phase. Both KSH components were found to be essential for pathogenesis. The separate $k s h A$ and $k s h B$ deletion mutants were unable to survive in mice models, and in macrophages, in the early stage of infection as well as in the chronic phase. These mutant strains, unlike wild type $M$. tuberculosis, were unable to catabolize

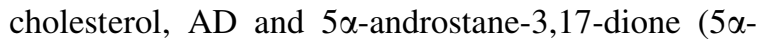
$\mathrm{AD})$. The $k s h B$ deletion mutant was also impaired in biosynthesis of penta-acylated trehalose (PAT), a glycolipid located at the surface of the cell wall. The data suggests that $\mathrm{KshB}$ can serve as a reductase for different oxygenases (Hu et al. 2010). 


\section{Engineering of Rieske non-heme oxygenase enzymes}

The essential role of KSH in the pathogenicity of $M$. tuberculosis as well as its potential in production of pharmaceutically interesting steroids make these enzymes an interesting target for protein engineering. One of the best studied ROs is biphenyl dioxygenase. Mutational engineering of this class III oxygenase (Batie et al. 1991) has resulted in enhanced activities, changed and/or expanded substrate ranges and altered regio selectivity (Furukawa et al. 2004 (review)). This susceptibility for enzyme engineering of biphenyl dioxygenase is in agreement with the finding that enzyme homologues from a Burkholderia strain and a Pseudomonas strain only differ by 20 amino acids, of a total of $\sim 460$ amino acid residues, while showing clear differences in functionality. Indeed, recombination of the genes encoding these highly similar enzymes by DNA shuffling resulted in altered substrate ranges and enhanced activities (Kumamaru et al. 1998). Intriguingly, the 5 KshA homologues of $R$. rhodochrous DSM43269 (Petrusma et al. 2011), as well as the KshA of $M$. tuberculosis (Capyk et al. 2009), all perform C9 $\alpha$ hydroxylation of steroid molecules while sharing $\sim 60 \%$ amino acid identity. However, all amino acid residues predicted to interact with the polycyclic ring structure of the substrate bound in the active site (Fig. 5) are conserved among known KshAs (Capyk et al. 2009). The KshA $A_{\text {DSM43269 homologues were all }}$ found to act on 3-ketosteroids showing overlap in substrate ranges, but with differences in substrate preference (Petrusma et al. 2011).

Naphthalene 1,2-dioxygenase of Pseudomonas sp. strain NCIB 9816-4 also has been well studied and subjected to several mutagenesis studies. This RO uses a large range $(>60)$ of aromatic compounds as substrates and performs different types of reactions, i.e. di- and monooxygenation, desaturation, sulphoxidation. The suitability of this RO for enzyme engineering was demonstrated by the fact that several point mutations around the active site affected substrate specificity as well as regio- and enantioselectivity (Ferraro et al. 2006; Parales 2003 (review)). The amino acid sequence identity between KshA, biphenyl dioxygenase and naphthalene 1,2-dioxygenase however is very low. Carbazole 1,9 $\alpha$-dioxygenase (CARDO, Janthinobacterium sp. strain J3) shares about $18 \%$ amino acid sequence identity with KshAs of both $R$. rhodochrous DSM43269 and M. tuberculosis and this RO is classified as a class III oxygenase on the basis of the electron transport chain enzymes (Nojiri et al. 2005). Point mutations of residues lining the substrate binding pocket resulted in CARDO mutants displaying changes in substrate specificity. Most of these mutated residues are situated near the active site. However, mutation of a residue near the entrance of the substrate binding pocket, close to a loop situated at the entrance of the substrate binding pocket, was found to improve activity on specific substrates (Uchimura et al. 2008). Similar loop regions are situated at the entrance of the substrate binding domain of many ROs. It has been speculated that this feature is involved in accommodation of substrates in the active site (Ferraro et al. 2005 (review)). Indeed, a study on chimeric KshA enzymes of $R$. rhodochrous DSM43269, with exchange of regions of the catalytic domain of two KshA homologues with different substrate preferences showed that the loop region, located at the entrance of the active site, strongly influences substrate specificity (Petrusma et al. 2012). A docking experiment with KshA of M. tuberculosis and 3-oxo-23,24-bisnorchola-1,4-dien-22-oyl-coenzyme A thioester (1,4-BNC-CoA) as substrate indicated the importance of amino acid residues in a pocket located at the entrance of the active site, which is the predicted location of the CoA group of this preferred substrate. In contrast to the amino acid residue at the active site that interacts with the polycyclic ring structure of ADD (Capyk et al. 2009), the residues near the entrance of the active site interacting with the CoA group are not conserved among known KshAs, indicative for differences in substrate range and specificity of the different KshAs (Capyk et al. 2011).

Detailed insights into the structure and function relationships of KshA, and the amino acid residues involved in substrate range and specificity, as well as regioselectivity of the hydroxylation, will provide a firm basis for subsequent rational engineering of KshA enzymes to improve synthesis of desired bioactive steroids. Also, since KSH was found to be essential for pathogenicity of M. tuberculosis (Hu et al. 2010) such insights may allow structure-based design of inhibitors against KSH activity to combat tuberculosis. 


\section{Conclusions}

Knowledge on $\mathrm{KSH}$ has increased substantially in recent years, paving the way for biotechnological exploitation of their catabolic potential. $\mathrm{KSH}$ is a key enzyme in bacterial steroid catabolism and therefore of strong physiological importance for a wide range of sterol degrading bacteria. Mutant bacterial strains devoid of KSH activity may convert relatively cheap sterols into bioactive steroids that are pharmaceutically interesting. KSH enzymes also are essential for pathogenicity of the notorious pathogen $M$. tuberculosis. Knowledge about the KSH catalysed reaction may allow design of specific enzyme inhibitors to combat tuberculosis. In summary, KSH enzymes are highly interesting for many biotechnological and medical applications. Further research will focus on (a) the regulation of their expression in bacterial hosts, against the background of other enzymes involved in sterol conversion, aiming to increase efficiency of metabolic conversions, (b) enzyme structural elements determining substrate/product specificity, followed by enzyme engineering to improve enzymatic conversions.

Open Access This article is distributed under the terms of the Creative Commons Attribution License which permits any use, distribution, and reproduction in any medium, provided the original author(s) and the source are credited.

\section{References}

Andor A, Jekkel A, Hopwood DA, Jeanplong F, Ilkoy E, Kónya A, Kurucz I, Ambrus G (2006) Generation of useful insertionally blocked sterol degradation pathway mutants of fast-growing mycobacteria and cloning, characterization, and expression of the terminal oxygenase of the 3-ketosteroid 9alpha-hydroxylase in Mycobacterium smegmatis mc2155. Appl Environ Microbiol 72:6554-6559

Arima K, Nagasawa M, Bae M, Tamura M (1969) Microbial transformation of sterols. Part I. Decomposition of cholesterol by microorganisms. Agric Biol Chem 33:1636-1643

Arnell R, Johannisson R, Lindholm J, Fornstedt T, Ersson B, Ballagi A, Caldwell K (2007) Biotechnological approach to the synthesis of 9alpha-hydroxylated steroids. Prep Biochem Biotechnol 37:309-321

Barry SM, Challis GL (2013) Mechanism and catalytic diversity of rieske non-heme iron-dependent oxygenases. ACS Catal 3(10) (Review). doi:10.1021/cs400087p

Batie CJ, Ballou DP, Correll CJ (1991) Phthalate dioxygenase reductase and related flavin-iron-sulfur containing electron transferases. In: Müller F (ed) Chemistry and biochemistry of flavoenzymes. CRC Press, Boca Raton, pp 543-556

Beharry ZM, Eby DM, Coulter ED, Viswanathan R, Neidle EL, Phillips RS, Kurtz DM Jr (2003) Histidine ligand protonation and redox potential in the Rieske dioxygenases: role of a conserved aspartate in anthranilate 1,2-dioxygenase. Biochemistry 42:13625-13636

Boyd DR, Sharma ND, Byrne BE, Haughey SA, Kennedy MA, Allen CC (2004) Dioxygenase-catalysed oxidation of alkylaryl sulfides: sulfoxidation versus cis-dihydrodiol formation. Org Biomol Chem 2:2530-2537

Bragin EY, Shtratnikova VY, Dovbnya DV, Schelkunov MI, Pekov YA, Malakho SG, Egorova OV, Ivashina TV, Sokolov SL, Ashapkin VV, Donova MV (2013) Comparative analysis of genes encoding key steroid core oxidation enzymes in fast-growing Mycobacterium spp. strains. J Steroid Biochem Mol Biol 138:41-53

Breskvar K, Cresnar B, Plaper A, Hudnik-Plevnik T (1991) Localization of the gene encoding steroid hydroxylase cytochrome P-450 from Rhizopus nigricans inside a Hin$d$ III fragment of genomic DNA. Biochem Biophys Res Commun 178:1078-1083

Bruijnincx PC, van Koten G, Klein Gebbink RJ (2008) Mononuclear non-heme iron enzymes with the 2-His-1-carboxylate facial triad: recent developments in enzymology and modeling studies. Chem Soc Rev 37:2716-2744 (Review)

Capyk JK, Eltis LD (2012) Phylogenetic analysis reveals the surprising diversity of an oxygenase class. J Biol Inorg Chem 17:425-436

Capyk JK, D'Angelo I, Strynadka NC, Eltis LD (2009) Characterization of 3 ketosteroid $9 \alpha$-hydroxylase, a Rieske oxygenase in the cholesterol degradation pathway of Mycobacterium tuberculosis. J Biol Chem 284:9937-9946

Capyk JK, Casabon I, Gruninger R, Strynadka NC, Eltis LD (2011) Activity of 3-ketosteroid $9 \alpha$-hydroxylase (KshAB) indicates cholesterol side chain and ring degradation occur simultaneously in Mycobacterium tuberculosis. J Biol Chem 286:40717-40724

Chakraborty J, Ghosal D, Dutta A, Dutta TK (2012) An insight into the origin and functional evolution of bacterial aromatic ring-hydroxylating oxygenases. J Biomol Struct Dyn 30:419-436

Correll CC, Batie CJ, Ballou DP, Ludwig ML (1992) Phthalate dioxygenase reductase: a modular structure for electron transfer from pyridine nucleotides to $[2 \mathrm{Fe}-2 \mathrm{~S}]$. Science 258:1604-1610

Costa S, Giovannini PP, Fantin G, Medici A, Pedrini P (2013a) New 9,10-secosteroids from biotransformations of hyodeoxycholic acid with Rhodococcus spp. Helv Chim Acta 96:1062

Costa S, Giovannini PP, Fantin G, Medici A, Pedrini P (2013b) New 9,10-Secosteroids from biotransformations of bile acids with Rhodococcus ruber. Helv Chim Acta 96:2124

Donova MV (2007) Transformation of steroids by actinobacteria: a review. Appl Biochem Microbiol 43:1-14 (Review)

Donova MV, Egorova OV (2012) Microbial steroid transformations: current state and prospects. Appl Microbiol Biotechnol 94:1423-1447 (Review)

Dresen C, Lin LY, D'Angelo I, Tocheva EI, Strynadka N, Eltis LD (2010) A flavin-dependent monooxygenase from 
Mycobacterium tuberculosis involved in cholesterol catabolism. J Biol Chem 285:22264-22270

Driscoll MD, McLean KJ, Levy C, Mast N, Pikuleva IA, Lafite P, Rigby SE, Leys D, Munro AW (2010) Structural and biochemical characterization of Mycobacterium tuberculosis CYP142: evidence for multiple cholesterol 27-hydroxylase activities in a human pathogen. J Biol Chem 285:38270-38282

Fernandes P, Cruz A, Angelova B, Pinheiro HM, Cabral JMS (2003) Microbial conversion of steroid compounds: recent developments. Enzyme Microb Technol 32:688-705 (Review)

Ferraro DJ, Gakhar L, Ramaswamy S (2005) Rieske business: structure-function of Rieske non-heme oxygenases. Biochem Biophys Res Commun 338:175-190 (Review)

Ferraro DJ, Okerlund AL, Mowers JC, Ramaswamy S (2006) Structural basis for regioselectivity and stereoselectivity of product formation by naphthalene 1,2-dioxygenase. J Bacteriol 188:6986-6994

Friemann R, Ivkovic-Jensen MM, Lessner DJ, Yu CL, Gibson DT, Parales RE, Eklund H, Ramaswamy S (2005) Structural insight into the dioxygenation of nitroarene compounds: the crystal structure of nitrobenzene dioxygenase. J Mol Biol 348:1139-1151

Furukawa K, Suenaga H, Goto M (2004) Biphenyl dioxygenases: functional versatilities and directed evolution. J Bacteriol 186:5189-5196 (Review)

Gibson DT, Wang KC, Sih CJ, Whitlock H Jr (1966) Mechanisms of steroid oxidation by microorganisms. IX. On the mechanism of ring A cleavage in the degradation of 9,10seco steroids by microorganisms. J Biol Chem 241:551559

Harayama S, Kok M, Neidle EL (1992) Functional and evolutionary relationships among diverse oxygenases. Annu Rev Microbiol 46:565-601 (Review)

Hegg EL, Que L Jr (1997) The 2-His-1-carboxylate facial triadan emerging structural motif in mononuclear non-heme iron(II) enzymes. Eur J Biochem 250:625-629 (Review)

Holland HL (1999) Recent advances in applied and mechanistic aspects of the enzymatic hydroxylation of steroids by whole-cell biocatalysts. Steroids 64:178-186 (Review)

Horinouchi M, Hayashi T, Kudo T (2012) Steroid degradation in Comamonas testosteroni. J Steroid Biochem Mol Biol 129:4-14

Hu Y, van der Geize R, Besra GS, Gurcha SS, Liu A, Rohde M, Singh M, Coates A (2010) 3-Ketosteroid 9alpha-hydroxylase is an essential factor in the pathogenesis of Mycobacterium tuberculosis. Mol Microbiol 75:107-121

Itagaki E, Hatta T, Wakabayashi T, Suzuki K (1990) Spectral properties of 3-ketosteroid-delta 1-dehydrogenase from Nocardia corallina. Biochim Biophys Acta 1040:281-286

Iyer LM, Koonin EV, Aravind L (2001) Adaptations of the helix-grip fold for ligand binding and catalysis in the START domain superfamily. Proteins 43:134-144

Jiang H, Parales RE, Gibson DT (1999) The alpha subunit of toluene dioxygenase from Pseudomonas putida F1 can accept electrons from reduced FerredoxinTOL but is catalytically inactive in the absence of the beta subunit. Appl Environ Microbiol 65:315-318

Kano S, Tanaka K, Hibino S, Shibuya S (1979) New synthesis of cortico steroids from 17-ketosteroids: application and stereochemical study of the unsaturated sulfoxide-sulfenate rearrangement. J Org Chem 44:1582-1584

Karlsson A, Parales JV, Parales RE, Gibson DT, Eklund H, Ramaswamy S (2003) Crystal structure of naphthalene dioxygenase: side-on binding of dioxygen to iron. Science 299:1039-1042

Klink M, Brzezinska M, Szulc I, Brzostek A, Kielbik M, Sulowska Z, Dziadek J (2013) Cholesterol oxidase is indispensable in the pathogenesis of Mycobacterium tuberculosis. PLoS One 8:e73333. doi:10.1371/journal. pone. 0073333

Knol J, Bodewits K, Hessels GI, Dijkhuizen L, van der Geize R (2008) 3-Keto-5alpha-steroid delta(1)-dehydrogenase from Rhodococcus erythropolis SQ1 and its orthologue in Mycobacterium tuberculosis $\mathrm{H} 37 \mathrm{Rv}$ are highly specific enzymes that function in cholesterol catabolism. Biochem J 410:339-346

Kumamaru T, Suenaga H, Mitsuoka M, Watanabe T, Furukawa K (1998) Enhanced degradation of polychlorinated biphenyls by directed evolution of biphenyl dioxygenase. Nat Biotechnol 16:663-666

Kweon O, Kim SJ, Baek S, Chae JC, Adjei MD, Baek DH, KimY C, Cerniglia CE (2008) A new classification system for bacterial Rieske non-heme iron aromatic ring-hydroxylating oxygenases. BMC Biochem 9:11

Lack NA, Yam KC, Lowe ED, Horsman GP, Owen RL, Sim E, Eltis LD (2010) Characterization of a carbon-carbon hydrolase from Mycobacterium tuberculosis involved in cholesterol metabolism. J Biol Chem 285:434-443

Lee K, Brand JM, Gibson DT (1995) Stereospecific sulfoxidation by toluene and naphthalene dioxygenases. Biochem Biophys Res Commun 212:9-15

Locher HH, Leisinger T, Cook AM (1991) 4-Sulphobenzoate 3,4-dioxygenase. Purification and properties of a desulphonative two-component enzyme system from Comamonas testosteroni $\mathrm{T}-2$. Biochem J 274:833-842

Madyastha KM, Joseph T (1993) Studies on the 14 alphahydroxylation of progesterone in Mucor piriformis. J Steroid Biochem Mol Biol 45:563-569

Mahato SB, Garai S (1997) Advances in microbial steroid biotransformation. Steroids 62:332-345 (Review)

Malaviya A, Gomes J (2008) Androstenedione production by biotransformation of phytosterols. Bioresour Technol 99:6725-6737 (Review)

Mason JR, Cammack R (1992) The electron transport proteins of hydroxylating bacterial dioxygenases. Ann Rev Microbiol 46:277-305 (Review)

Mathieu JM, Mohn WW, Eltis LD, LeBlanc JC, Stewart GR, Dresen C, Okamoto K, Alvarez PJ (2010) 7-Ketocholesterol catabolism by Rhodococcus jostii RHA1. Appl Environ Microbiol 76:352-355

McLeod MP, Warren RL, Hsiao WW, Araki N, Myhre M, Fernandes C, Miyazawa D, Wong W, Lillquist AL, Wang D, Dosanjh M, Hara H, Petrescu A, Morin RD, Yang G, Stott JM, Schein JE, Shin H, Smailus D, Siddiqui AS, Marra MA, Jones SJ, Holt R, Brinkman FS, Miyauchi K, Fukuda M, Davies JE, Mohn WW, Eltis LD (2006) The complete genome of Rhodococcus sp. RHA1 provides insights into a catabolic powerhouse. Proc Natl Acad Sci USA 103:15582-15587

Meena LS, Rajni (2010) Survival mechanisms of pathogenic Mycobacterium tuberculosis H37Rv. FEBS J 277:24162427 (Review) 
Megges R, Müller-Frohne M, Pfeil D, Ruckpaul K (1990) In: Ruckpaul K, Rein H (eds) Microbial steroid hydroxylation enzymes in glucocorticoid production. Akademie Verlag, Berlin (Review)

Mohn WW, Wilbrink MH, Casabon I, Stewart GR, Liu J, van der Geize R, Eltis LD (2012) Gene cluster encoding cholate catabolism in Rhodococcus spp. J Bacteriol 194:6712-6719

Nagasawa M, Bae M, Tamura G, Arima K (1969) Microbial transformation of sterols. II. Cleavage of sterol side chains by microorganisms. Agric Biol Chem 33:1644-1650

Nakatsu CH, Straus NA, Wyndham RC (1995) The nucleotide sequence of the Tn5271 3-chlorobenzoate 3,4-dioxygenase genes (cbaAB) unites the class IA oxygenases in a single lineage. Microbiology (UK) 141:485-495

Nam JW, Nojiri H, Yoshida T, Habe H, Yamane H, Omori T (2001) New classification system for oxygenase components involved in ring-hydroxylating oxygenations. Biosci Biotechnol Biochem 65:254-263

Neidig ML, Solomon EI (2005) Structure-function correlations in oxygen activating non-heme iron enzymes. Chem Commun (Camb). 47:5843-5863 (Review)

Neidle EL, Hartnett C, Ornston LN, Bairoch A, Rekik M, Harayama S (1991) Nucleotide sequences of the Acinetobacter calcoaceticus benABC genes for benzoate 1,2dioxygenase reveal evolutionary relationships among multicomponent oxygenases. J Bacteriol 173:5385-5395

Nesbitt NM, Yang X, Fontán P, Kolesnikova I, Smith I, Sampson NS, Dubnau E (2010) A thiolase of Mycobacterium tuberculosis is required for virulence and production of androstenedione and androstadienedione from cholesterol. Infect Immun 78:275-282

Nojiri H, Nam JW, Kosaka M, Morii KI, Takemura T, Furihata K, Yamane H, Omori T (1999) Diverse oxygenations catalyzed by carbazole 1,9a-dioxygenase from Pseudomonas sp. Strain CA10. J Bacteriol 181:3105-3113

Nojiri H, Ashikawa Y, Noguchi H, Nam JW, Urata M, Fujimoto Z, Uchimura H, Terada T, Nakamura S, Shimizu K, Yoshida T, Habe H, Omori T (2005) Structure of the terminal oxygenase component of angular dioxygenase, carbazole 1,9 $\alpha$-dioxygenase. J Mol Biol 351:355-370

Ouellet H, Guan S, Johnston JB, Chow ED, Kells PM, Burlingame AL, Cox JS, Podust LM, de Montellano PR (2010) Mycobacterium tuberculosis CYP125A1, a steroid C27 monooxygenase that detoxifies intracellularly generated cholest-4-en-3-one. Mol Microbiol 77:730-742

Pandey AK, Sassetti CM (2008) Mycobacterial persistence requires the utilization of host cholesterol. Proc Natl Acad Sci USA 105:4376-4380

Parales RE (2003) The role of active-site residues in naphthalene dioxygenase. J Ind Microbiol Biotechnol 30:271-278 (Review)

Parales RE, Parales JV, Gibson DT (1999) Aspartate 205 in the catalytic domain of naphthalene dioxygenase is essential for activity. J Bacteriol 181:1831-1837

Peterson DH, Murray HC (1952) Microbiological oxygenation of steroids at carbon-11. J Am Chem Soc 74:1871-1872

Petrusma M (2011) Exploring 3-ketosteroid 9 $\alpha$-hydroxylases of Rhodococcus rhodochrous DSM43269: biochemical, physiological, molecular and structural characterization of a key enzyme in bacterial steroid degradation. Dissertation, University of Groningen
Petrusma M, Dijkhuizen L, van der Geize R (2009) Rhodococcus rhodochrous DSM 43269 3-ketosteroid $9 \alpha-$ hydroxylase, a two-component iron-sulfur-containing monooxygenase with subtle steroid substrate specificity. Appl Environ Microbiol 75:5300-5307

Petrusma M, Hessels GI, Dijkhuizen L, van der Geize R (2011) Multiplicity of 3-ketosteroid-9 $\alpha$-hydroxylase enzymes in Rhodococcus rhodochrous DSM43269 for the specific degradation of different classes of steroids. J Bacteriol 193:3931-3940

Petrusma M, Dijkhuizen L, van der Geize R (2012) Structural features in the KshA terminal oxygenase protein determining substrate preference of 3-ketosteroid 9 $\alpha$-hydroxylase enzymes. J Bacteriol 194:115-121

Pinto A, Tarasev M, Ballou DP (2006) Substitutions of the "bridging" aspartate 178 result in profound changes in the reactivity of the Rieske center of phthalate dioxygenase. Biochemistry 45:9032-9041

Rengarajan J, Bloom BR, Rubin EJ (2005) Genome-wide requirements for Mycobacterium tuberculosis adaptation and survival in macrophages. Proc Natl Acad Sci USA 102:8327-8332

Resnick SM, Gibson DT (1993) Biotransformation of anisole and phenetole by aerobic hydrocarbonoxidizing bacteria. Biodegradation 4:195-203

Rosłoniec KZ, Wilbrink MH, Capyk JK, Mohn WW, Ostendorf M, van der Geize R, Dijkhuizen L, Eltis LD (2009) Cytochrome P450 125 (CYP125) catalyses C26-hydroxylation to initiate sterol side-chain degradation in Rhodococcus jostii RHA1. Mol Microbiol 74:1031-1043

Sassetti CM, Rubin EJ (2003) Genetic requirements for mycobacterial survival during infection. Proc Natl Acad Sci USA 100:12989-12994

Schnappinger D, Ehrt S, Voskuil MI, Liu Y, Mangan JA, Monahan IM, Dolganov G, Efron B, Butcher PD, Nathan C, Schoolnik GK (2003) Transcriptional adaptation of Mycobacterium tuberculosis within macrophages: insights into the phagosomal environment. J Exp Med 198:693-704

Sih CJ, Tai HH, Tsong YY, Lee SS, Coombe RG (1968) Mechanisms of steroid oxidation by microorganisms. XIV. Pathway of cholesterol side-chain degradation. Biochemistry 7:808-818

Söhngen NL (1913) Benzin, Petroleum, Paraffino1 and Paraffin als Kohlenstoff-und Energiequelle fur Mikroben. Zentbl Bakt Parasitenk (Abt. II) 37:595

Strijewski A (1982) The steroid-9 alpha-hydroxylation system from Nocardia species. Eur J Biochem 128:125-135

Suzuki K, Sanga K, Chikaoka Y, Itagaki E (1993) Purification and properties of cytochrome $\mathrm{P}-450$ (P-450lun) catalyzing steroid 11 beta-hydroxylation in Curvularia lunata. Biochim Biophys Acta 1203:215-223

Sydor PK, Barry SM, Odulate OM, Barona-Gomez F, Haynes SW, Corre C, Song L, Challis GL (2011) Regio- and stereodivergent antibiotic oxidative carbocyclizations catalysed by Rieske oxygenase-like enzymes. Nat Chem 3:388-392

Tarasev M, Pinto A, Kim D, Elliott SJ, Ballou DP (2006) The "bridging" aspartate 178 in phthalate dioxygenase facilitates interactions between the Rieske center and the iron(II)-mononuclear center. Biochemistry 45:10208-10216

Tarasev M, Kaddis CS, Yin S, Loo JA, Burgner J, Ballou DP (2007) Similar enzymes, different structures: phthalate 
dioxygenase is an alpha3alpha3 stacked hexamer, not an alpha3beta3 trimer like "normal" Rieske oxygenases. Arch Biochem Biophys 466:31-39

Torok DS, Resnick SM, Brand JM, Cruden DL, Gibson DT (1995) Desaturation and oxygenation of 1,2-dihydronaphthalene by toluene and naphthalene dioxygenase. J Bacteriol 177:5799-5805

Uchimura H, Horisaki T, Umeda T, Noguchi H, Usami Y, Li L, Terada T, Nakamura S, Shimizu K, Takemura T, Habe H, Furihata K, Omori T, Yamane H, Nojiri H (2008) Alteration of the substrate specificity of the angular dioxygenase carbazole 1,9 $\alpha$-dioxygenase. Biosci Biotechnol Biochem 72:3237-3248

Van der Geize R, Dijkhuizen L (2004) Harnessing the catabolic diversity of rhodococci for environmental and biotechnological applications. Curr Opin Microbiol 7:255-261 (Review)

Van der Geize R, Hessels GI, van Gerwen R, Vrijbloed JW, van Der Meijden P, Dijkhuizen L (2000) Targeted disruption of the kstD gene encoding a 3-ketosteroid delta(1)-dehydrogenase isoenzyme of Rhodococcus erythropolis strain SQ1. Appl Environ Microbiol 66:2029-2036

Van der Geize R, Hessels GI, van Gerwen R, van der Meijden P, Dijkhuizen L (2001) Unmarked gene deletion mutagenesis of $k s t D$, encoding 3-ketosteroid $\Delta^{1}$-dehydrogenase, in Rhodococcus erythropolis SQ1 using $\operatorname{sacB}$ as counterselectable marker. FEMS Microbiol Lett 205:197-202

Van der Geize R, Hessels GI, Van Gerwen R, Van der Meijden P, Dijkhuizen L (2002) Molecular and functional characterization of kshA and $\mathrm{kshB}$, encoding two components of 3-ketosteroid $9 \alpha$-hydroxylase, a class IA monooxygenase, in Rhodococcus erythropolis strain SQ1. Mol Microbiol 45:1007-1018

Van der Geize R, Yam K, Heuser T, Wilbrink MH, Hara H, Anderton MC, Sim E, Dijkhuizen L, Davies JE, Mohn WW, Eltis LD (2007) A gene cluster encoding cholesterol catabolism in a soil actinomycete provides insight into Mycobacterium tuberculosis survival in macrophages. Proc Natl Acad Sci USA 104:1947-1952

Van der Geize R, Hessels GI, Nienhuis-Kuiper M, Dijkhuizen L (2008) Characterization of a second Rhodococcus erythropolis SQ1 3-ketosteroid 9alpha-hydroxylase activity comprising a terminal oxygenase homologue, KshA2, active with oxygenase-reductase component KshB. Appl Environ Microbiol 74:7197-7203

Wang FQ, Yao K, Wei DZ (2011) From soybean phytosterols to steroid hormones. Soybean Health. InTech-Open Access Publisher, Rijeka, pp 232-252 (Review)

Wang PH, Lee TH, Ismail W, Tsai CY, Lin CW, Tsai YW, Chiang YR (2013) An oxygenase-independent cholesterol catabolic pathway operates under oxic conditions. PLoS One 8:e66675

Wilbrink MH, Petrusma M, Dijkhuizen L, van der Geize R (2011) FadD19 of Rhodococcus rhodochrous DSM43269: a steroid-CoA ligase essential for degradation of C24branched sterol side chains. Appl Environ Microbiol 77:4455-4464

Yam KC, D'Angelo I, Kalscheuer R, Zhu H, Wang JX, Snieckus V, Ly LH, Converse PJ, Jacobs WR Jr, Strynadka N, Eltis LD (2009) Studies of a ring-cleaving dioxygenase illuminate the role of cholesterol metabolism in the pathogenesis of Mycobacterium tuberculosis. PLoS Pathog 5(e100 0344):5

Yam KC, Okamoto S, Roberts JN, Eltis LD (2011) Adventures in Rhodococcus-from steroids to explosives. Can J Microbiol 57:155-168 (Review)

Yang X, Gao J, Smith I, Dubnau E, Sampson NS (2011) Cholesterol is not an essential source of nutrition for Mycobacterium tuberculosis during infection. J Bacteriol 193:1473-1476 